\title{
Fullfield neutron microscopy based on refractive optics
}

Leemreize, Hanna; Knudsen, Erik B.; Birk, Jonas O.; Strobl, Markus; Detlefs, Carsten; Poulsen, Henning F.

Published in:

Journal of Applied Crystallography

Link to article, DOI:

$10.1107 / \mathrm{s} 1600576719012858$

Publication date:

2019

Document Version

Publisher's PDF, also known as Version of record

Link back to DTU Orbit

Citation (APA):

Leemreize, H., Knudsen, E. B., Birk, J. O., Strobl, M., Detlefs, C., \& Poulsen, H. F. (2019). Fullfield neutron microscopy based on refractive optics. Journal of Applied Crystallography, 52(6).

https://doi.org/10.1107/s1600576719012858

\section{General rights}

Copyright and moral rights for the publications made accessible in the public portal are retained by the authors and/or other copyright owners and it is a condition of accessing publications that users recognise and abide by the legal requirements associated with these rights.

- Users may download and print one copy of any publication from the public portal for the purpose of private study or research.

- You may not further distribute the material or use it for any profit-making activity or commercial gain

- You may freely distribute the URL identifying the publication in the public portal 


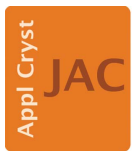

JOURNAL OF

APPLIED

CRYSTALLOGRAPHY

ISSN 1600-5767

Received 12 June 2019

Accepted 17 September 2019

Edited by A. Borbély, Ecole National Supérieure des Mines, Saint-Etienne, France

Keywords: neutron imaging; neutron diffraction; small-angle neutron scattering; SANS; stress mapping; grain mapping; neutron optics; compound refractive lenses.

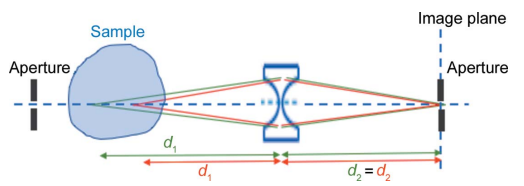

C 2019 International Union of Crystallography

\section{Full-field neutron microscopy based on refractive optics}

\author{
Hanna Leemreize, ${ }^{\mathrm{a}, \mathrm{b}}$ Erik B. Knudsen, ${ }^{\mathrm{a}}$ Jonas O. Birk, ${ }^{\mathrm{b}}$ Markus Strobl, ${ }^{\mathrm{c}}$ Carsten \\ Detlefs $^{\mathrm{d}}$ and Henning F. Poulsen ${ }^{\mathrm{a} *}$
}

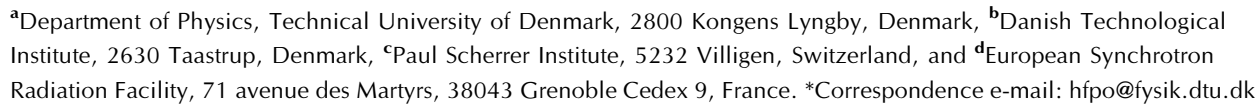

Placing a compound refractive lens (CRL) as an objective in a neutron beam generates new possibilities for $2 \mathrm{D}$ and $3 \mathrm{D}$ nondestructive mapping of the structure, strain and magnetic domains within extended objects. A condenser setup is introduced that allows correction for the lateral chromatic aberration. More generally, for full-field microscopy the loss in performance caused by the chromatic aberration can be more than offset by introducing arrays of CRLs and exploiting the fact that the field of view can be much larger than the physical aperture of the CRL. Comments are made on the manufacture of such devices. The potential use is illustrated by comparisons between state-of-the-art instrumentation and suggested approaches for bright-field microscopy, smallangle neutron scattering microscopy, grain mapping and mapping of stresses. Options are discussed for depth-resolved imaging inspired by confocal light microscopy. Finally, experimental demonstrations are given of some of the basic properties of neutron full-field imaging for a single CRL.

\section{Introduction}

Neutron imaging is a rapidly expanding field (Lehmann, 2015; Kardjilov et al., 2018; Treimer, 2019). The relatively low brilliance of neutron sources means that imaging experiments typically require the use of a polychromatic beam with a relatively large divergence, and this is particularly true for $3 \mathrm{D}$ and time-resolved studies. With such beams, it is natural to base bright-field imaging studies on placing a $2 \mathrm{D}$ detector downstream of but in close proximity to the sample. This approach enables a large variety of contrast to be explored, including attenuation contrast (Kallmann, 1948; Strobl et al., 2009), phase contrast (Strobl et al., 2008) and more specialized techniques such as spectral imaging (Lehmann et al., 2014), Bragg edge contrast for mapping of stresses (Santisteban et al., 2001) and phases (Steuwer et al., 2004; Woracek et al., 2014), extinction contrast for mapping of large grains (Cereser et al., 2017), and some versions of polarized neutron imaging for visualizing magnetic field distributions (Sales et al., 2019) and magnetic domains (Kardjilov et al., 2008; Schulz et al., 2010; Strobl et al., 2019; Jorba et al., 2019).

One basic limitation, though, is that the resolution deteriorates with larger sample-volume-detector distances, unless one compromises on the divergence of the incoming beam. This necessitates a trade-off between spatial resolution and time resolution, and might limit spatial resolution for extended objects and in particular where extensive sample auxiliaries are required.

Likewise, for imaging the scattering from a sample propagating at an angle to the optical axis of the incoming beam 
(known in classical optics as dark-field imaging) this approach of positioning the detector in close proximity to the sample does not work. For small-angle scattering contrast (Strobl et al., 2017) and diffraction-based imaging (Woracek et al., 2018), distance is required to obtain the required angular resolution. Spatial discrimination is then obtained by inserting slits, collimators or phase grids into the beam path, and mapping is provided by scanning the sample.

The classical optical approach to imaging at a distance is to use lenses. A range of optical devices have been demonstrated for focusing neutron beams based on neutron guides, capillary optics (Chen et al., 1992), Fresnel zone plates (Altissimo et al., 2004), compound refractive lenses (CRLs; Eskildsen et al., 1998), Wolter optics (Mildner \& Gubarev, 2011) and sextupole magnets (Suzuki et al., 2004). Evidently, spatial mapping can be made with such focused beams by placing the sample in the focal plane and scanning it with respect to the beam. However, this is a tedious approach, and for time-resolved work the fact that different sub-volumes of the sample are probed at different times can be troublesome. Another solution is to place the sample downstream of the focal spot and generate a magnified image by projection microscopy. In both cases the spatial resolution is ultimately limited by the focal spot size.

Neutron full-field microscopy is an alternative. Here, the entire volume of interest in the sample is illuminated and an objective lens is introduced between the sample and the detector. As always for classical microscopy, an inverted and potentially magnified or demagnified image forms in the image plane. Using CRLs, proof-of-concept studies have been presented (Beguiristain et al., 2002) and it has been demonstrated that the objective lens can discriminate against scattered neutrons to enable absorption contrast imaging from hydrogenous objects (Cremer et al., 2005). To our knowledge, however, full-field neutron microscopy has been applied only infrequently, if at all, presumably because only a fraction of the neutrons in the incoming beam are used, leading to problems with the signal-to-noise $(\mathrm{S} / \mathrm{N})$ ratio. In contrast, CRLs have seen extensive use at synchrotron sources (Snigirev et al., 1996) as condensers and objectives and for hard X-ray microscopes (Simons et al., 2015).

In this paper we revisit the concept of a neutron microscope with CRL-type objectives. In contrast with the synchrotron case, we show that such a neutron CRL can often be described as a lens with no attenuation, and with a resulting field of view which is much larger than the physical aperture. With this insight we discuss the optimization of lens design. Next, we propose imaging configurations that optimize the throughput, i.e. the fraction of neutrons in the primary beam that contribute to the final image. We introduce the concepts of a kinoform lens and of a CRL array: an optics system comprising a 2D grid of CRLs with either parallel or diverging optical axes. Likewise, we discuss solutions to overcome the issue of chromatic aberration. The focus is primarily on the design of the objective, as an optimization of the incoming beam may involve other types of optics, such as supermirror-based focusing.

First we present optical properties for candidate materials for CRL production and briefly discuss the feasibility of
Table 1

Comparison of CRL candidate materials.

$\rho$ is density. $\delta$ is the refractive index increment. $\sigma_{\text {inc }}$ and $\sigma_{\text {abs }}$ are the incoherent and the absorption cross sections, respectively [listed by Sears (1992)] and $\mu$ is the linear attenuation coefficient. The numbers for $\mathrm{C}$ represent diamond. The first row refers to $\mathrm{X}$-rays of $17 \mathrm{keV}$ energy, while data for the neutron setups in the rows below relate to a wavelength of $4 \AA$.

\begin{tabular}{llllll}
\hline & $\rho\left(\mathrm{g} \mathrm{cm}^{-3}\right)$ & $\delta \times 10^{-6}$ & $\sigma_{\text {inc }}($ barn $)$ & $\sigma_{\text {abs }}($ barn $)$ & $\mu\left(\mathrm{m}^{-1}\right)$ \\
\hline X-rays, Be & 1.85 & 1.25 & & & 46 \\
$\mathrm{Be}$ & 1.85 & 24.4 & 0.0018 & 0.0076 & 0.12 \\
$\mathrm{C}$ & 3.51 & 29.3 & 0.001 & 0.0035 & 0.08 \\
$\mathrm{MgF}_{2}$ & 3.15 & 12.6 & 0.08 & 0.065 & 0.44 \\
$\mathrm{Bi}^{208} \mathrm{~Pb}$ & 9.78 & 5.95 & 0.0084 & 0.038 & 0.13 \\
$\mathrm{SiO}_{2}$ & 11.3 & 7.79 & 0 & 0.00048 & $<0.01$ \\
\hline
\end{tabular}

manufacturing them. Next, we introduce analytical expressions for the relevant optical parameters and define a figure of merit (FOM) for the overall efficiency of the imaging system. Following the presentation of several new optics concepts aimed at improving the FOM, we illustrate the potential use of CRL-based neutron microscopy by discussing solutions for bright-field microscopy, depth-resolved imaging, small-angle neutron scattering (SANS) microscopy, grain mapping and mapping of local strain from powder diffraction signals. Finally, we demonstrate some of the basic properties of neutron full-field imaging for a single CRL in an experiment performed on the BOA test instrument at the Paul Scherrer Institute (PSI).

\section{Optical properties of a neutron CRL and imaging system}

\subsection{Candidate materials}

Candidate materials for neutron CRLs have a large $\delta / \mu$ ratio, where $\delta$ is the refractive index decrement and $\mu$ is the linear attenuation coefficient, as calculated from the incoherent and absorption cross sections, $\sigma_{\text {inc }}$ and $\sigma_{\text {abs }}$, respectively (Eskildsen et al., 1998). Moreover, the materials should be crystalline to avoid an otherwise massive attenuation due to the coherent cross sections $\sigma_{\text {coh }}$ (all practical CRL materials have $\sigma_{\text {coh }} \gg \sigma_{\text {inc }}+\sigma_{\text {abs }}$ ). A selection of candidate materials is presented in Table 1. Oxides and fluorides of $\mathrm{Be}, \mathrm{Bi}$ and $\mathrm{Pb}$ are particularly interesting. In the following, $\mathrm{Be}$ is treated as a reference material, as it has superior properties for neutron CRLs and, at the same time, lenses with very high accuracy are commercially available, as they are the preferred material for synchrotron use. However, Be is difficult to machine and existing lenses are costly.

Among the other materials, crystals of $\mathrm{MgF}_{2}$ (Eskildsen et al., 1998) and $\mathrm{SiO}_{2}$ (Gähler et al., 1980) are easily available and can be machined by traditional milling machines. We argue that the resulting accuracy of approximately $5 \mu \mathrm{m}$ will in general be sufficient for spatial resolutions in the range 20$1000 \mu \mathrm{m}$. Moreover, we speculate that CRLs of ${ }^{208} \mathrm{~Pb}$ (or natural $\mathrm{Pb}$ ) and $\mathrm{Bi}$ can be cast, CRLs in graphite can be made by powder compacting, and lenses in diamond can be made by 
laser ablation. In all cases, spurious effects such as small-angle scattering would have to be characterized and process optimization may be necessary.

With so many materials and technologies at hand, we argue that batch production of neutron CRLs with complicated 3D shapes should be possible, and discuss methodologies that require such configurations.

It is instructive to compare with the X-ray case. Comparing the tabulated numbers in the first line of Table 1 with the rest, it is apparent that the $\delta / \mu$ ratio is more favourable for neutrons, and that the attenuation is at least two orders of magnitude smaller for neutrons. This implies that a neutron CRL for the same focal length and physical aperture is much closer to the classical thin lens behaviour, i.e. the transmission of the lens will be limited by the physical aperture rather than by attenuation in the lens material.

\subsection{Geometry of a single neutron CRL}

A comprehensive treatment of X-ray and neutron CRLs is presented by Cremer (2012). Here we will apply a formalism that has been developed for the hard X-ray microscope at ESRF, as this introduces equations for chromatic aberration.

The geometry is illustrated in Fig. 1. We shall consider a two-dimensional CRL with $N$ identical parabolic shaped lenslets, with a distance $T$ between centres, each with a radius of curvature $R$ and a physical thickness $T_{\mathrm{p}} \leq T$. The focal length of each lenslet is then $f=R /(2 \delta)$. The physical aperture is given by the diameter $2 Y=2\left(R T_{\mathrm{p}}\right)^{1 / 2}$, and we shall assume neutrons at distances to the optical axes that are larger than $2 Y$ to be absorbed by circular slits placed at the entry and exit
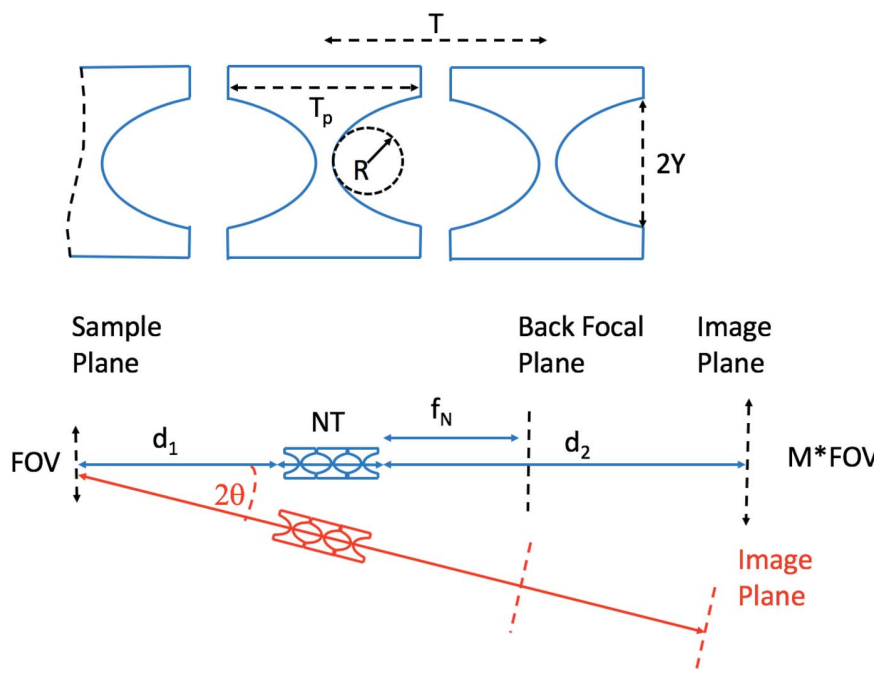

Figure 1

(Top) The geometry of a neutron compound refractive lens with $N$ lenslets. The distance between lenslets, $T$, the physical thickness of each lenslet, $T_{\mathrm{p}}$, the radius of curvature at the apex, $R$, and the physical aperture, $2 Y$, are marked. (Bottom) The geometry of bright-field (blue) and diffraction-based (red) neutron microscopy. $2 \theta$ is the scattering angle, $d_{1}$ the distance from the sample plane to the entry point of the objective, $d_{2}$ the distance from the exit point of the objective to the image plane and $f_{N}$ the focal length of the objective. $M$ and FOV symbolize the magnification and field of view, respectively. of the CRL. The focal length of the entire CRL is $f_{N}$ and the numerical aperture is NA (as defined by the FWHM).

The geometry of the imaging setup is also illustrated in Fig. 1. The magnification $M$ and the field of view FOV in the sample (as defined by the FWHM) are determined by the distances $d_{1}$ and $d_{2}$.

The relevant optical parameters can all be determined by geometric optics, and more specifically by the use of ray tracing by means of, for example, the ray matrix transfer formalism (Cremer, 2012; Poulsen et al., 2014; Simons et al., 2017) or by Monte Carlo methods (Willendrup et al., 2014). For reference use we provide analytical expressions for the optical parameters for some general and special cases. In the thin lens case, with $N T \ll f_{N}$, we have

$$
f_{N}=f / N \quad \text { and } \quad M=\frac{d_{2}}{d_{1}} .
$$

The same equations for the general thick lens case are derived by Simons et al. (2017):

$$
\begin{gathered}
f_{N}=f \varphi \cot (N \varphi), \\
M=\frac{1}{\cos (N \varphi)} \frac{f_{N}}{d_{1}-f_{N}}=\cos (N \varphi) \frac{d_{2}-f_{N}}{f_{N}} .
\end{gathered}
$$

Here $\varphi=(T / f)^{1 / 2}$. For $T=T_{\mathrm{p}}$ this leads to $f \varphi=Y /(2 \delta)^{1 / 2}$.

Next we define the transparent lens case as the (thin or thick lens) case where the attenuation of the neutrons within the parabolic part is negligible. Key optical properties are then given by the dimensions of the entire CRL, that is by the physical aperture $2 Y$ and by the length of the CRL NT. We have $N \varphi=(2 \delta)^{1 / 2} N T_{\mathrm{p}} / Y T$, where the last factor is typically close to 1 . Moreover,

$$
\mathrm{NA}=\min \left[\frac{2 Y}{d_{1}} \cos (N \varphi), 2(2 \delta)^{1 / 2} \sin (N \varphi)\right] .
$$

The first term reflects the limitation of the CRL as a collimator and the second term its limitation in terms of refractive power. The cosine factor in the first term originates from the fact that the neutron trajectory within the CRL is approximately sinusoidal with period $2 \pi N \varphi$ (Poulsen et al., 2017). Next, we have

$$
\mathrm{FOV}=2 Y \cos (N \varphi)\left(\frac{d_{1}}{N T}+1\right) .
$$

We also define the opaque lens case as the (thin or thick lens) case where the attenuation is so strong that NA and FOV are determined by the parabolic section of the lens, and $2 Y$ can therefore be deemed irrelevant. The relevant equations are provided by Poulsen et al. (2017):

$$
\begin{gathered}
\mathrm{NA}=2.35 \delta \frac{M}{M+1}\left(\frac{2 N}{\mu R}\right)^{1 / 2}, \\
\mathrm{FOV}=2.35^{2} \frac{2 \delta}{\mu} \frac{1}{\mathrm{NA}}\left[(N \varphi)^{2}-\sin ^{2}(N \varphi)\right]^{-1 / 2} .
\end{gathered}
$$


Table 2

Comparison of key optical properties for a CRL objective with lenslets of radius $R=50 \mu \mathrm{m}$ and thickness $T=2 \mathrm{~mm}$ for a focal length of $f_{N}=25 \mathrm{~cm}$ and a magnification of $M=10$.

The first row refers to $\mathrm{X}$-rays of $17 \mathrm{keV}$ energy, while data for the neutron setups in the rows below relate to a wavelength of $4 \AA$. $N$ is the number of lenslets. The three numbers listed for the numerical aperture (NA) and the field of view (FOV) correspond to the FWHM for the transparent lens formalism [equations (4) and (5)], the FWHM calculated by the opaque lens formalism [equations (6) and (7)], and the results of McStas simulations. The smaller values are marked in bold type.

\begin{tabular}{|c|c|c|c|c|c|c|c|c|}
\hline \multirow{2}{*}{\multicolumn{2}{|c|}{$N$}} & \multirow[b]{2}{*}{$\begin{array}{l}N T \\
(\mathrm{~mm})\end{array}$} & \multicolumn{3}{|c|}{ NA (mrad) } & \multicolumn{3}{|c|}{ FOV $(\mathrm{mm})$} \\
\hline & & & $\begin{array}{l}\text { Trans- } \\
\text { parent }\end{array}$ & Opaque & McStas & $\begin{array}{l}\text { Trans- } \\
\text { parent }\end{array}$ & Opaque & McStas \\
\hline $\mathrm{X}$-rays, Be & 71 & 142 & 2.5 & 0.68 & - & 1.3 & 1.7 & - \\
\hline $\mathrm{Be}$ & 4 & 8 & 2.5 & 16 & 2.97 & 21 & 1900 & 19.5 \\
\hline $\mathrm{C}$ & 3 & 6 & 2.5 & 18 & 3.02 & 25 & 3100 & 22.7 \\
\hline $\mathrm{MgF}_{2}$ & 8 & 16 & 2.5 & 12 & 2.92 & 11 & 380 & 9.9 \\
\hline $\mathrm{Bi}$ & 17 & 34 & 2.5 & 8 & 2.79 & 5 & 720 & 4.7 \\
\hline${ }^{208} \mathrm{~Pb}$ & 13 & 26 & 2.5 & 9 & 2.75 & 7 & 1225 & 6.1 \\
\hline $\mathrm{SiO}_{2}$ & 9 & 18 & 2.5 & 11 & 2.89 & 10 & & 7.88 \\
\hline
\end{tabular}

The depth of field (DOF) for neutrons is defined exclusively by geometric optics. In terms of the FWHM we have (for all lens cases)

$$
\mathrm{DOF}=\frac{y_{\mathrm{s}}}{\mathrm{NA}}
$$

Here $y_{\mathrm{s}}=y_{\mathrm{d}} / M$ is the spatial resolution in the sample plane, while $y_{\mathrm{d}}$ is the resolution in the imaging plane.

In Table 2, X-ray and neutron numbers for NT, NA and FOV are compared for a specific setting that has been frequently used for hard X-ray microscopy on beamline ID06 at ESRF (Simons et al., 2015, 2018). It appears that the NA values for the neutron cases are 3.5 times larger than for the $\mathrm{X}$-ray case, while the FOV is 5-20 times larger. Moreover, the attenuation, which at most is $\exp (-\mu N T / 3)$, is very small in the neutron case. (The tabulated values are for cold neutrons, as these are relevant for most of the applications presented. We have $\delta / \mu \propto \lambda$, where $\lambda$ is the neutron wavelength.)

Finally, the transparent lens case is seen to be a good approximation for neutron work for the chosen geometric setting. For all materials the NA is underestimated by $10-20 \%$ in comparison with McStas simulations (Willendrup et al., 2019), and the FOV is correspondingly overestimated by a similar amount.

\subsection{Chromatic aberration}

Given the relatively large NAs of neutron CRLs, the intrinsic optical limit to the spatial resolution given by the diffraction theorem is below $100 \mathrm{~nm}$ and as such is truly negligible. In the following we shall assume that aberrations due to manufacturing errors of the lenses are also small. [For neutrons, the spatial resolution is in fact often so relatively poor that the lenses can be manufactured with a spherical profile; the associated spherical aberration is negligible (Cremer, 2012).] The spatial resolution will then be given by the chromatic aberration, the detector pixel size and the $\mathrm{S} / \mathrm{N}$ ratio. Let the FWHM of the blurring function associated with chromatic aberration be defined by a radius $r$, as measured in the sample plane. In the thin lens limit we have

$$
r=\frac{M+1}{M} Y \epsilon
$$

where $\epsilon=|\Delta E / E|$ is the relative energy bandwidth (FWHM).

In the true thick lens limit where the physical aperture does not play a role, the relevant equation for the root-meansquare (RMS) of the spread in the sample plane is (Poulsen et al., 2017)

$$
\begin{gathered}
\sigma_{\mathrm{chr}}=\frac{\sigma_{\mathrm{a}} \epsilon_{\mathrm{RMS}} d_{\mathrm{chr}}}{M}, \\
d_{\mathrm{chr}}=N \varphi\left(\frac{d_{1} d_{2}}{f \varphi}-f \varphi\right) \cos (N \varphi) \\
+\left[\frac{d_{1} d_{2}}{f \varphi}+f \varphi+N \varphi\left(d_{1}+d_{2}\right)\right] \sin (N \varphi),
\end{gathered}
$$

where $\sigma_{\mathrm{a}}=\mathrm{NA} / 2.35$ is the RMS value for the numerical aperture [equation (6)] and $\epsilon_{\mathrm{RMS}}=\epsilon / 2.35$.

The chromatic aberration is clearly a critical issue for highspatial-resolution bright-field imaging work, but less so for low-resolution work of relevance, e.g. in diffraction-based microscopy. As an example, for a physical aperture of $2 Y=$ $5 \mathrm{~mm}$ and a magnification of $M=1$ the energy bandwidth can be $0.4,4$ and $40 \%$ for a spatial resolution (FWHM) of $10 \mu \mathrm{m}$, $100 \mu \mathrm{m}$ and $1 \mathrm{~mm}$, respectively.

For bright-field microscopy it is actually possible to correct the lateral chromatic aberrations by focusing a condenser on the entry plane of the objective if the incident divergence is smaller than the NA. A very successful demonstration of this principle for hard X-rays was performed by Falch et al. (2016).

Using geometric optics, the sample is assumed to be weakly scattering such that only absorption contrast is detected. In this context wide-angle scattering, e.g. as in extinction contrast, is indistinguishable from absorption contrast, as the scattered radiation does not enter the objective lens. Within this framework it is possible to find a relation between the position where a ray intersects the sample and its direction that minimizes the chromatic aberration in the detector plane. It turns out that the best solution is to focus the incident beam onto the entry plane of the CRL (Falch et al., 2016). An improvement in resolution can only be achieved, however, when the random divergence of the incident beam is smaller than the NA of the CRL.

A general solution will require the construction of a neutron achromat. Poulsen et al. (2014) proposed to create such a component by combining a converging Fresnel zone plate and a diverging CRL. Using ray tracing it was shown that a variation of much less than $1 \%$ in focal length can be achieved over a 10\% energy band width (Poulsen et al., 2014), but this solution is associated with several challenges. Firstly, to the knowledge of the authors it is difficult to match the two types of optics in terms of $Y$, NA and FOV. Moreover, the transmission of the achromat will not be close to 1 , as is the case for the CRL on its own. Finally, generalizing the 
achromat solution to a $2 \mathrm{D}$ array is challenging. We shall not consider this achromat solution further in this article.

\subsection{Back focal plane}

As for any lens, a neutron CRL is associated with a Fourier plane placed at a distance $f_{N}$ from the exit of the CRL. There is a linear relation between the coordinates $\left(y_{\mathrm{B}}, z_{\mathrm{B}}\right)$ in this plane and the angles $\left(\xi_{y}, \xi_{z}\right)$ in the sample plane (Poulsen et al., 2018):

$$
y_{\mathrm{B}}=\frac{f_{N}}{\cos (N \varphi)} \xi_{y} \quad \text { and } \quad z_{\mathrm{B}}=\frac{f_{N}}{\cos (N \varphi)} \xi_{z} .
$$

Here $\xi_{y}$ is the projection of the angle between a ray and the optical axis onto the $y$ axis and $\xi_{z}$ is the projection onto the $z$ axis.

With focal lengths of order $1 \mathrm{~m}$, an angular offset of $1 \mathrm{mrad}$ corresponds to $1 \mathrm{~mm}$ in the back focal plane. Hence, the Fourier space resolution obtainable can be excellent.

\subsection{Figure of merit for efficiency}

Different science cases call for different compromises between spatial, angular and time resolution. In this paper the focus is on providing extensive 2D maps of a given spatial and angular resolution as fast as possible. The generalization to three dimensions is foreseen to be via tomographic reconstruction methods. For bright-field imaging an alternative is depth-resolved methods (Section 4.2), while for diffractionbased imaging (at scattering angles not too close to 0 or $\pi$ ) an alternative is layer-by-layer reconstruction.

For a given spatial resolution in the sample, $s$, we introduce the figure of merit FOM,

$$
\mathrm{FOM}=\left(\frac{\mathrm{FOV}}{s}\right)^{2} \mathrm{NA}^{2} \epsilon(r<s) \Theta_{\mathrm{CRL}} \Theta_{\mathrm{Det}}(M) .
$$

The first term defines the number of positions in the sample plane imaged simultaneously, the second the angular range covered, and the third the maximum energy bandwidth and therefore the maximum chromatic aberration error that is acceptable given the spatial resolution. $\Theta_{\mathrm{CRL}}$ is the average transmission of the CRL and $\Theta_{\text {Det }}$ is the quantum efficiency of the detector. We require the pixel size of the detector to be substantially smaller than $M s$.

For a bright-field experiment, ideally the field of view matches the sample area of interest, the NA matches the divergence of the incoming beam and $\epsilon$ matches the energy bandwidth provided, while $\Theta_{\mathrm{CRL}}=\Theta_{\text {Det }}=1$.

\subsection{Design of a single neutron CRL}

In the transparent lens limit it follows from equations (4) and (3) that

$$
2 Y=\mathrm{NA} d_{1} \frac{1}{\cos (N \varphi)}=\mathrm{NA} f_{N} \frac{1+M \cos (N \varphi)}{M \cos (N \varphi)^{2}} .
$$

As stated above, the maximum NA for a CRL is $2(2 \delta)^{1 / 2}$ and requires large magnifications. Hence, given constraints on laboratory space $\left(f_{N}<1 \mathrm{~m}\right)$ it is generally speaking impractical to make a neutron CRL with a physical aperture $2 Y$ larger than about $8 \mathrm{~mm}$.

Next, reducing the empty space between lenslets is favourable for all properties, and we shall in the rest of this section assume $T=T_{\mathrm{p}}$. Then for the FOV in the combined transparent and thin lens limit we have

$$
\mathrm{FOV}=4 \delta \frac{f_{N}^{2}}{Y}\left(\frac{M+1}{M}\right)
$$

Hence, with increasing physical aperture, the FOV decreases. Moreover, the total thickness increases and the chromatic aberration radius also increases [equation (9)]. In terms of the FOM as defined in equation (13), it appears that the smallaperture configuration is preferable. As a result we have FOV $2 Y$ ( $c f$. also Table 2). The imaging setup is then essentially a camera obscura setup with a lens inserted at the pinhole. The insertion of a lens improves the spatial resolution.

From the equations it also follows that, for specified $f_{N}$ and $M$, the optical parameters and FOM do not depend directly on $R$ and $T_{\mathrm{p}}$ but only on the product $\left(R T_{\mathrm{p}}\right)^{1 / 2}$. In terms of design, the optimal choice of $R$ and $T=T_{\mathrm{p}}$ is therefore defined by manufacturing issues. This is also the case in the thick lens limit (Poulsen et al., 2017).

\section{Improving the efficiency \\ 3.1. Kinoform lenses}

A well known solution to increasing the physical aperture of a lens for a given focal length is to use a 'kinoform' profile (Jordan et al., 1970). Whereas the profile of each lens element in a CRL (see Fig. 1) was assumed to be parabolic in order to provide a phase shift $\Delta \phi(r)=(2 \pi \delta / R) r^{2}$, in a kinoform lens the phase shift is taken modulo $m 2 \pi(m \in \mathbb{N})$, resulting in a stepped profile with step height $\Delta z_{m}=m \lambda / \delta$ as shown in Fig. 2 .

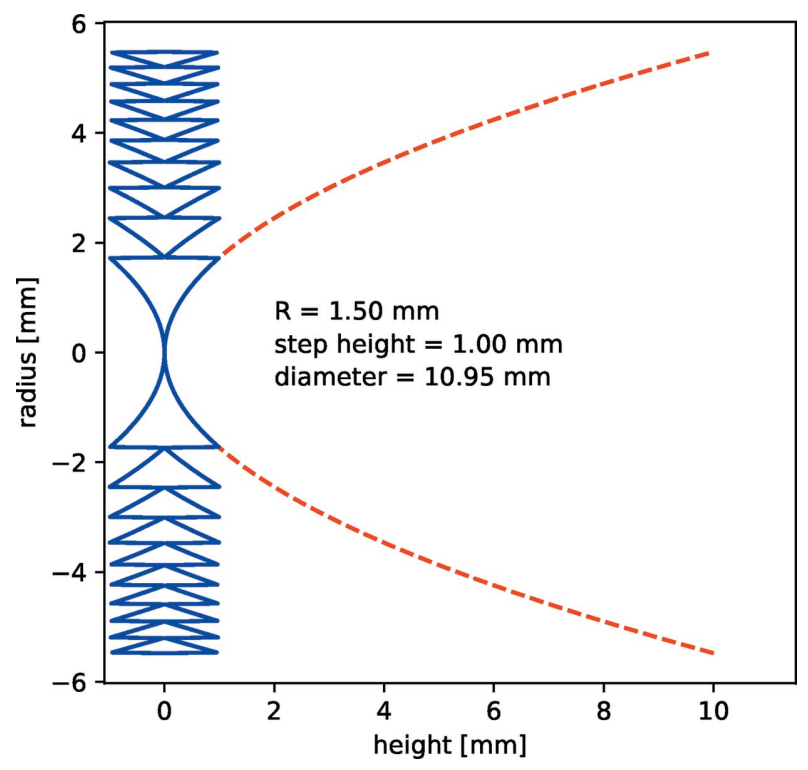

Figure 2

A sketch of a kinoform lens. The first ten zones are marked. 
The kinoform lens will provide an increased NA for a reduced thickness, at the expense of an increased problem with chromatic aberration [equation (9)]. However, the introduction of steps in the lens profile also has disadvantages. The phase difference across the step can be made exactly $m 2 \pi$ at only one wavelength, due to the direct dependence of $z_{m}$ on $\lambda$ and the dispersion of the lens material, $\delta(\lambda)$. The error is cumulative, such that the zones will become progressively out of phase with the zeroth-zone beam. Furthermore, manufacturing limitations will introduce errors at each step. As the zones become more and more narrow away from the centre, the useful part (sufficiently far away from the inside and outside step) of the zone shrinks and eventually vanishes. As the steps and their associated manufacturing errors may introduce artefacts and distortions in a direct image, we speculate that the main use of kinoform lenses will be as condensers and beam-shaping elements.

\subsection{Design of a $2 \mathrm{D}$ array of CRLs}

An obvious way to increase the FOM is to apply a number of CRLs in parallel, positioned in a $2 \mathrm{D}$ grid of say $N \times N$ grid points. We shall call the resulting optical device a CRL array. It is of interest to use the same detector for several grid points, for reasons of cost and size of detector frame. Likewise, it is of interest to manufacture the CRL array as one entity, which is pre-aligned, rather than $N \times N$ independent CRLs to be aligned individually. As discussed in Section 2.1, there are several potential solutions to manufacture such complex optics.
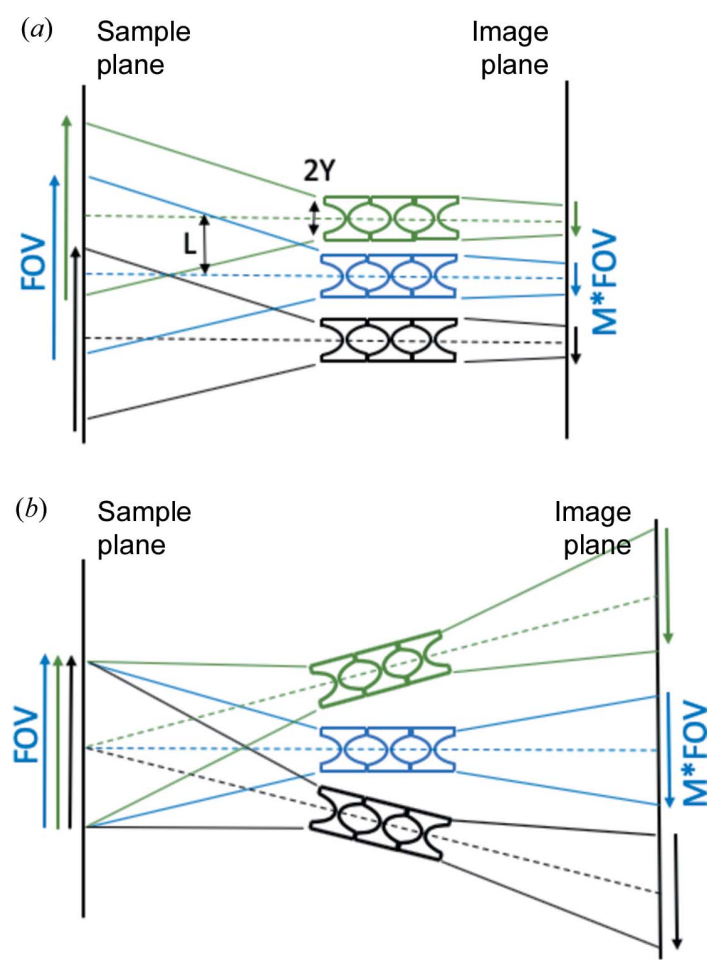

Figure 3

(a) A CRL array with parallel optical axes. (b) A CRL array with all optical axes coinciding in the sample plane. FOV is the field of view and $M$ is the magnification.
Two examples of the configuration of a CRL array are illustrated in Fig. 3:

(i) Parallel optical axes of the CRLs. Let the distance between the centres of the holes be $L$ [Fig. 3(a)]. If we place the CRLs as close together as possible we have $L \simeq 2 Y$. For $M \geq 1$ and FOV $\gg 2 Y$, it appears that a superposition of subimages will take place in the image plane. These sub-images would have to be either disentangled or modelled as part of a forward projection algorithm. ${ }^{\mathbf{1}}$ For sparse images, typically related to diffraction-based (dark-field) microscopy, this may be feasible. However, for classical attenuation or phasecontrast imaging the added complexity is hardly merited.

For $L \simeq 2 Y$ and $M<2 Y / F O V$ there will be no superposition. Hence, by demagnifying the image one may sample both spatial and angular degrees of freedom almost fully. However, this comes at the expense of smaller detector pixels (and is therefore potentially associated with an inferior detector quantum efficiency) and a larger total number of pixels. Moreover, for a high degree of demagnification $(M \ll 1)$ the chromatic aberration causes the resolution to deteriorate [equation (9)].

(ii) Divergent optical axes. The manufacturing principles outlined above would allow for the various individual CRLs to have different optical axes. For some applications it may be favourable for the optical axes of the various CRLs to coincide at the origin on the sample plane [Fig. 3(b)]. In that way one can approach a $4 \pi$ coverage of the scattering from a given volume. The condition for a complete spatial and angular sampling is now for $L \simeq 2 Y$ and

$$
\frac{M}{1+M}<\frac{2 Y}{\mathrm{FOV}}
$$

This still comes at the cost of an increased number of pixels. In practice, the compromise between a large FOV, a large NA and small chromatic aberrations may lead to designs where the grid spacing $L$ is substantially larger than $2 Y$, in which case the areas on the CRL array not participating in the focusing should be masked. Generally speaking, the CRL array concept complies well with the introduction of such masks.

\section{Applications}

In the following we aim to illustrate potential applications by comparing the FOM of CRL-based full-field microscopy solutions with the FOM of state-of-the-art instruments. Throughout, we shall consider CRLs based on Be and neglect the efficiency of the detectors, as for the sake of simplicity we assume these to be the same.

\subsection{Bright-field imaging}

One obvious application is for imaging in cases involving bulky sample environments. As an example, consider imaging with polarized neutrons, where the polarization analyser

\footnotetext{
${ }^{1}$ Within the CRL the path of a ray is sinusoidal. For small $Y$ a non-inverted 1:1 image can be obtained and then the sub-images can be merged. However, the NA would be small and the $\mathrm{S} / \mathrm{N}$ ratio therefore poor.
} 
equipment requires an extended distance of, say, $0.5 \mathrm{~m}$ between the sample and the detector. As typical parameters for such measurements today (Strobl et al., 2019) we shall consider imaging of a sample of dimension $2 \mathrm{~cm}$. Using an energy bandwidth of $3 \%$ (given by the spin precession resolution), a spatial resolution of $500 \mu \mathrm{m}$ is achievable, corresponding to a divergence of the incoming beam of $1 \mathrm{mrad}$. Correspondingly, FOM $=(0.02 \mathrm{~m} / 0.0005 \mathrm{~m})^{2} \times 1 \mathrm{mrad}^{2} \times$ $0.03=4.8 \times 10^{-5} \mathrm{rad}^{2}$.

A microscope setup with a sample-to-detector distance of $3 \mathrm{~m}$ and a 1:1 magnification leaves ample space to put the polariser either between the sample and the objective or between the objective and the detector. Using the thin lens formalism [equations (4) and (9)], a single CRL with $N=60$ Be lenslets with $R=2 \mathrm{~mm}$ and $T=8 \mathrm{~mm}$ would have $f_{N}=$ $0.52 \mathrm{~m}, d_{1}=1.04 \mathrm{~m}, 2 Y=8 \mathrm{~mm}, \mathrm{FOV}=2.5 \mathrm{~cm}, N T=0.48 \mathrm{~m}$, $\mathrm{NA}=8 \mathrm{mrad}$ and $\epsilon=3 \%$. For this we have $\mathrm{FOM}=(0.02 \mathrm{~m} /$ $0.0005 \mathrm{~m})^{2} \times 8 \mathrm{mrad}^{2} \times 0.03=0.0030 \mathrm{rad}^{2}$. Hence, for the specified spatial resolution we have a gain of 65 . Additional increases are possible using the achromat condenser and/or an array of identical CRLs configured in the fashion of Fig. 3(b).

Another potential use is for in-line phase-contrast imaging (Allman et al., 2000) or alternatively for removing unwanted edge-contrast effects in attenuation-contrast images (Strobl et al., 2008). Notably, the objective defines a specific plane perpendicular to the optical axis, the sample plane (Fig. 1). Placing the exit edge of the sample on the sample plane will result in a pure attenuation image with no superposed fringes from phase contrast. Alternatively, by translating the sample along the optical axis, pure in-line phase-contrast images can be obtained at any requested Fresnel number. Given the weak interaction of matter with thermal neutrons, the single image (one distance) algorithm presented by Paganin et al. (2002) for reconstructing both the phase and the amplitude can be expected to work. This was recently corroborated by applications in neutron tomography by Paganin et al. (2019).

\subsection{Depth-resolved microscopy}

In bio-imaging, confocal microscopy is a powerful tool for mapping specimens in three dimensions without the use of sample rotations and tomographic reconstruction algorithms (Tiziani \& Uhde, 1994). The large NA of classical light microscopy does in itself provide a depth resolution, but the emitted light from the out of focus layers reduces the quality of the image. In confocal microscopy the spurious light from these out of focus layers is much reduced. The drawback is that the method in its original form only provides information about one local volume at a time, and as such requires scanning of the beam or the sample in $(x, y, z)$. Current implementations relax this constraint by using a layer beam or multiplexing the output in other ways.

The relatively large NA of a neutron CRL objective and the weak interaction of thermal neutrons with many materials open up a similar approach for neutron bright-field imaging. For many specimens, in particular biological materials, the coherent cross section is much larger than the cross section for absorption. As such, the use of an objective is a way of using the scattering signal to map the structure of the specimen, where the scattering acts as an internal source in the same way as the fluorescent signal from dyed molecules does in visiblelight microscopy. We speculate that neutrons from out of focus layers will again lead to a deterioration of the image. Transferring the principles of confocal microscopy to neutrons would then be a solution to improve image quality at the expense of data acquisition time.

A basic setup for neutron confocal microscopy is shown in Fig. 4 . The nominal neutron wavelength, $\lambda_{0}$, is associated with a sample plane and an image plane, defined by distances $d_{1}^{0}$ and $d_{2}$, respectively. The sample plane for a slightly different wavelength, $\lambda$, is at $d_{1}$. In the thin lens approximation we have

$$
\begin{aligned}
\frac{d_{1}-d_{1}^{0}}{d_{1}^{0}} & =\frac{M}{M+1}\left(\frac{\lambda_{0}^{2}}{\lambda^{2}}-1\right) \\
& \simeq-2 \frac{M}{M+1} \frac{\lambda-\lambda_{0}}{\lambda_{0}}
\end{aligned}
$$

Hence, various depths in a sample can be probed simultaneously if the setting allows energy determination with sufficient accuracy. As mentioned above, 3D mapping is then achieved by scanning the sample with respect to the beam in $(x, y)$.

As an example, consider an implementation where the incoming beam has dimensions of $50 \times 50 \mu \mathrm{m}, \mathrm{NA}=10 \mathrm{mrad}$, $f_{N}=0.25 \mathrm{~m}$ and the magnification is $M=1$. Then the depth of field is $5 \mathrm{~mm}$ [equation (8)]. This corresponds to a $2 \%$ change in the working distance $d_{1}$. According to equation (18) one can therefore generate a profile along the line with a resolution of $5 \mathrm{~mm}$, provided the energy discrimination of the setup is better than $2 \times 10^{-2}$, which is realistic for time-of-flight (TOF) operation. In a different embodiment one may use the condenser achromat described in Section 2.3. In this way, one only maps one point at a time, but the $\mathrm{S} / \mathrm{N}$ ratio is improved.

Point-by-point scanning is evidently tedious and, similar to the situation for visible light, a compromise is required between the suppression of signal from out of focus points and the data acquisition time. The use of a layered incoming beam

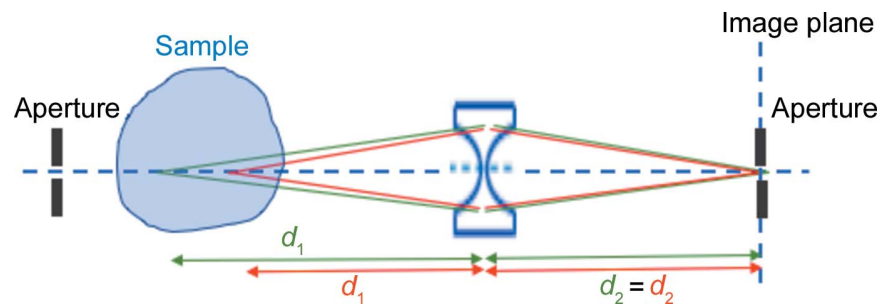

Figure 4

Basic setup for confocal neutron microscopy. The incoming beam is a pencil beam, defined by an aperture and/or a condenser. It defines a line through the sample. In connection with the imaging geometry, this defines one internal point in the sample and removes stray scattering from neighbouring parts of the sample. A detector is placed after the aperture. The imaging configurations for two energies (green and red) are shown. 
could be a place to start. Moreover, multiplexing can be obtained by using an objective array as in Fig. 3(a).

\subsection{SANS microscopy}

With X-rays, imaging of the small-angle X-ray scattering (SAXS) signal is a mature field. Scanning a sample with respect to a micro-beam is used for studies of anisotropic systems such as bone, wood and hair (Gourrier et al., 2007). Hard X-ray microscopy is a powerful alternative, used e.g. for providing 3D images of colloidal systems (Byelov et al., 2013). With neutrons, SANS imaging by means of rastering the sample is used regularly, for example to study flow (Penfold \& Tucker, 2007; Dewhurst \& Grillo, 2016). CRLs are also used in SANS setups as condensers with the detector in the back focal plane of the lens.

In Fig. 5 we sketch a SANS microscope. By adding CRLs this setup will be able to cover the entire $Q$ range of interest. There are no moving parts and the CRL array can be added to an existing SANS instrument. The $Q$ resolution of each CRL (at fixed wavelength) is approximately $\Delta Q=4 \pi \mathrm{NA} / 2 \lambda$. This is in the region of $0.01 \AA^{-1}$, which matches the $Q$ resolution of SANS instruments (Dewhurst \& Grillo, 2016). However, depending on the position within the sample plane there is an offset to the $Q$ range covered by a given CRL.

It appears that for FOV $<2 Y / M$ (no overlap between the images from neighbouring CRLs, $c f$. Section 3.2) essentially all the neutrons available for SANS are also available for SANS microscopy: one simply swaps between a setup with no spatial resolution and high $Q$ resolution (classical SANS) and one with medium spatial resolution and medium to high $Q$ resolution (SANS microscopy). Moreover, for studying one specific region in $Q$, one may increase the FOV to become larger than $2 Y / M$.

As an example of the current state of the art, we shall consider a rastering study of a mechanical pen performed on the D33 SANS instrument at the Institut Laue-Langevin, Grenoble, France (Dewhurst \& Grillo, 2016). For this a $200 \mu \mathrm{m}$ pinhole was inserted in front of the sample in a beam of $10 \%$ bandwidth and a divergence of $5 \mathrm{mrad}$. The SANS detector was positioned $15 \mathrm{~m}$ from the sample. The contrast in $Q$ in this particular case was defined by summing over the

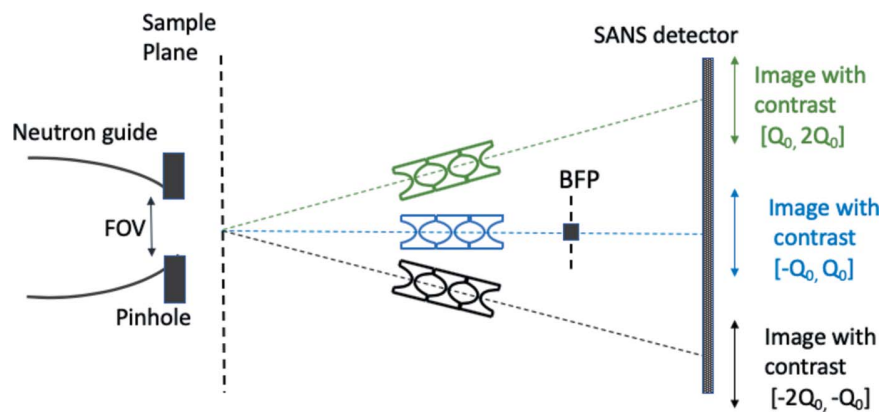

Figure 5

The proposed setup for SANS microscopy. The CRLs all have a field of view (FOV) that matches the dimension of the incoming beam. The beamstop in the back focal plane (BFP) of the central CRL is used to remove the direct beam. intensities in the area detector, resulting in three $Q$ intervals: low, medium and high $Q$. The low- $Q$ range corresponded to an angle (FWHM) of $10 \mathrm{mrad}$. Hence, the figure of merit of this setup is $\mathrm{FOM}=1^{2} \times(10 \mathrm{mrad})^{2} \times 0.1=1.0 \times 10^{-5} \mathrm{rad}^{2}$.

For the SANS microscope, let us first consider the low- $Q$ range with the same incoming beam but a pinhole of $30 \mathrm{~mm}$. We shall assume that this only changes the divergence of the incoming beam by a negligible amount. We require a similar spatial resolution of $200 \mu \mathrm{m}$. As an example of an implementation we consider a single Be CRL with $Y=R=T_{\mathrm{p}}=T=$ $0.0035 \mathrm{~m}, N=91$ and 1:1 imaging. According to the transparent lens case this will be associated with $f_{N}=0.70 \mathrm{~m}, N T=$ $0.32 \mathrm{~m}, \mathrm{NA}=4.1 \mathrm{mrad}, \mathrm{FOV}=30 \mathrm{~mm}$ and $\epsilon=2.8 \%$. The figure of merit of this setup is $\mathrm{FOM}=(0.03 / 0.0002)^{2} \times$ $(4.1 \mathrm{mrad})^{2} \times 0.028=0.0109 \mathrm{rad}^{2}$. Hence, the gain is a factor of 1000 . Note also that with the small incoming divergence a condenser achromat would be suitable.

For the higher $Q$ ranges we have to use a CRL array, and consequently we will reduce the FOV to $2 Y=7 \mathrm{~mm}$ to avoid the overlap of images in the image plane. The gain is then of the order of 50 .

Another potential application of SANS microscopy is the equivalent of tensor SAXS. Here, the anisotropy (or texture) of a small-angle scattering pattern is measured during a $2 \mathrm{D}$ tomography scan - sample rotations about two orthogonal axes are needed in order to determine the full tensor anisotropy. In the X-ray case a micro-beam is rastered across the sample, and a full SAXS pattern is recorded at each point (Liebi et al., 2015, 2018). We propose that equivalent data could be acquired by SANS microscopy using a lens array. A set of slits in the back focal plane could be used to improve the angular resolution, for example to select a relevant length scale. Each lens in the array then provides a real-space image corresponding to the range in azimuth collected by that particular lens. At least four lenses arranged in a ring are required for rank-2 tensor anisotropy, eight lenses for rank-4 etc.

\subsection{Phase and strain mapping}

Within materials science and geoscience it is of interest to map the crystalline phases in a sample in three dimensions and, for a given phase, to map the local texture and the strain tensor. The latter may be used for generation of a stress map. State-of-the-art instruments are monochromatic diffractometers such as STRESS-SPEC at FRM-II (Munich, Germany) (Hofmann et al., 2006, 2013), and TOF diffractometers like VULCAN at SNS (Oak Ridge, Tennessee, USA) (Wang et al., 2010), ENGIN-X at ISIS (Didcot, UK) (Santisteban et al., 2006) and the planned instrument BEER at ESS (Lund, Sweden) (Fenske et al., 2016). Using slits and radial collimators, these provide a large coverage of the scattering from one specified local volume. Mapping is then obtained by scanning the sample with respect to the beam in $(x, y, z)$.

The approach of a neutron dark-field microscope is instead to map the relevant (sub)volume of the sample in one setting with no movement or rotation of the sample. This may speed up the entire data acquisition, thereby supporting 
time-resolved measurements or making industrial exploration cheaper.

In this section we shall assume the diffracting elements within the sample to be sufficiently small that the scattered signal is that of a (textured) powder diffraction pattern. Moreover, we assume that the phases present are either known a priori or have been identified in other ways, e.g. from neutron powder diffraction on the same sample.

A CRL array with the optical axes coinciding in the sample plane [Fig. 3(b)] can in principle be used to obtain $4 \pi$ coverage of the scattering. Such a construction would comprise an inner shell of CRLs and an outer shell of detectors. This would be a very efficient collection of data.

Equally important, by providing projections from a number of directions, a 3D reconstruction can be obtained without any rotation of the sample. For this purpose a substantially reduced coverage is sufficient. The exact number of projections required and the optimal configuration will depend on the specifications for the microscope. To our knowledge, such an optimization has not been performed for a highly divergent white-beam source.

In practice, the merit of using a neutron dark-field microscope depends on the ratio between the FOV and the dimension of the gauge volume, $s$. For coarse mapping of large components on a centimetre scale or for weak neutron sources it is not relevant. Another concern is that the CRL performance deteriorates with increasing neutron energy.

In the following we consider an implementation at a monochromatic source. If we place a CRL with a circular aperture in the diffraction beam at a scattering angle of $2 \theta$, the resolution becomes $\Delta 2 \theta=\pi \mathrm{NA} / 4$ (FWHM). From this follows a strain resolution of

$$
\left|\frac{\Delta d}{d}\right|=\frac{\pi}{8} \cot (\theta) \text { NA. }
$$

For NA $=5 \mathrm{mrad}$, the resulting strain resolution at $2 \theta=\pi / 2$ is $2 \times 10^{-3}$. To determine the average strain (within a voxel in the sample) with a higher accuracy than the resolution, one may interpolate or fit a model of the 'peak profile' to intensities associated with neighbouring $2 \theta$ bins, similar to how neutron strain scanning is performed today. Moreover, the CRLs can be offset azimuthally. Notably, with an energy spread of say $3 \%$ and $M=1$, the chromatic aberration is not an issue for $s \geq 0.5 \mathrm{~mm}$ and $M \leq 0.1$ [equation (9)].

Hence, it appears that conceptually one can think of a darkfield microscope in terms of each of the individual radial collimators being replaced by a 1D stack of CRLs. To avoid overlap of images demagnification is required. The demagnification leads to long distances $d_{1}$ and a large thickness $N T$.

The advantage is that angular and spatial degrees of freedom are separated and one therefore can make a 2D map for each CRL. 2D mapping for a layer in the sample is then readily provided by using a line beam as the incoming beam, and $3 \mathrm{D}$ mapping by translating the sample with respect to the beam and stacking layers on top of each other. Moreover, the issue with pseudo peak shifts due to partially filled gauge volumes, which has been discussed at length for neutron strain instruments (Pirling, 2011), is overcome in this method. The substantial disadvantages are the overhead of constructing a voluminous and heavy 'bank' of CRLs and the fact that the 2D detector must have a larger FOV and be equipped with more pixels. Background will also become more of an issue.

To illustrate the potential of this apporach, we compare it with the state-of-the-art materials science diffractometer STRESS-SPEC at the FRM-II reactor (Hofmann et al., 2006, 2013). Operating at $2.3 \AA$ with an energy spread of $\epsilon=3 \%$, the smallest gauge volume is $(0.5 \mathrm{~mm})^{3}$. The corresponding radial collimator is placed at a distance of $15 \mathrm{~cm}$, and therefore has a horizontal divergence of $3.3 \mathrm{mrad}$. It is $35 \mathrm{~cm}$ long and covers the full detector height vertically and $9^{\circ}$ in $2 \theta$ horizontally.

As an implementation of a dark-field microscopy setup at $2.3 \AA$, consider initially a vertical array of 1D CRLs, in the configuration shown in Fig. 3(b). We specify a demagnification of $M=0.1$, and space availability leads to $d_{1}=2 \mathrm{~m}$. Let each CRL have $N=196$ lenslets and a relatively small physical aperture $Y=T=T_{\mathrm{p}}=R=1 \mathrm{~mm}$. As a result, $\mathrm{NA}=5 \mathrm{mrad}$ and $\mathrm{FOV}=14 \mathrm{~mm}$. With this setting the criterion of equation (14) is fulfilled, so overlap of sub-images is avoided. Moreover, let the horizontal width of these lenses be $6.6 \mathrm{~mm}$. With such a setting, a 1D vertical array can replace one slot in the radial collimator, providing the same angular coverage and resolution. However, information is now provided in parallel for $\mathrm{FOV} / s=28$ positions along the optical axis of the incoming beam instead of 1 . In practice the gain will be lower, due to transmission losses and a lower $\mathrm{S} / \mathrm{N}$ ratio.

In a more advanced embodiment, the 1D lenses above are replaced with $2 \mathrm{D}$ lenses with the same specifications (an NA of $5 \mathrm{mrad}$ in both directions) and the incoming beam is configured to be a line beam of dimensions $14 \times 0.5 \mathrm{~mm}$. In this way an entire area of $14 \times 14 \mathrm{~mm}$ in one layer of the sample is mapped in one setting. At the same time, the angular resolution in the $2 \theta$ direction is slightly improved to become $2.5 \mathrm{mrad}$.

\subsection{Mapping of grains and single crystals}

For a monochromatic and parallel neutron beam, crystalline elements of a certain volume give rise to distinct diffraction spots at specified angular settings. Placed on a goniometer, either the entire sample (in the case of a single crystal) or an embedded grain can be reoriented such that the direction of the diffracted beam is aligned with the optical axis of the objective. The situation is then fully equivalent to the hard X-ray dark-field microscopy setup implemented on beamline ID06 at ESRF (Poulsen et al., 2017; Simons et al., 2015, 2018). By scanning sample tilt angles, maps of the local orientation can be mapped. In a polychromatic setting, the axial strain can be mapped by shifts in the energy spectrum, similar to the process outlined in Section 4.4.

For a more complete coverage, to map say a grain with an internal degree of mosaicity larger than the NA, one may perform a continuous scan of a sample tilt stage while acquiring data and/or exploit the use of a CRL array. In this case the data are sparse, and overlap of images is less of an issue. 
The reciprocal-space resolution of a dark-field microscope for elastic scattering is described in detail by Poulsen et al. (2017). For neutrons the resolution function will tend to be an isotropic Gaussian, except for $2 \theta$ near 0 and $\pi$. For points off the optical axis, the centre point for the reciprocal-space resolution function shifts. Moreover, it should be noted that acquiring images while scanning one of the sample tilts will not ensure a uniform sampling of reciprocal space. Strategies for coping with these issues are discussed by Poulsen et al. (2017).

A prerequisite to using the dark-field microscope for grain mapping is that the grain and its orientation have been identified. Multigrain indexing methods similar to those developed for X-rays (Sørensen et al., 2012) are therefore required. Peetermans et al. (2014) and Cereser et al. (2017) described such methodologies for neutrons in a low-resolution and a TOF setting, respectively. Similarly to the X-ray case, there is a strong degree of complementarity between such tomographic methods, providing an overview of the entire specimen, and dark-field microscopy, focusing on only one grain at a time.

\section{Experimental demonstration}

A demonstration of bright-field neutron microscopy using a CRL as objective was performed using an ad hoc setup on the
BOA test instrument at PSI. Using a double-bounce pyrolytic graphite monochromator with a mosaicity of 100 arcminutes, a beam was created with an average wavelength of $4 \AA$ and an energy bandwidth of $3 \%$. From McStas simulations we estimate a divergence of the exit beam $\alpha_{\mathrm{v}}=7.4 \mathrm{mrad}$ (vertical) $\times$ $\alpha_{\mathrm{h}}=20 \mathrm{mrad}$ (horizontal). The sample was a $\mathrm{Gd}$ reference pattern consisting of a star with 36 spokes [see Fig. 6(b)]. The illuminated area was limited by absorbers in the setup but provided a near-uniform illumination.

The Be CRL was configured as follows: $N=4, R=50 \mu \mathrm{m}, T=$ $2 \mathrm{~mm}, T_{\mathrm{p}}=1 \mathrm{~mm}$ and hence $2 Y=0.45 \mathrm{~mm}$. To avoid scattering through the non-convex parts of the CRL, two Gd pinholes were placed at the entry and exit of the CRL. The same CRL was used for X-ray microscopy with a resulting spatial resolution of about $100 \mathrm{~nm}$. The 2D detector was a PSI midiboxtype scintillator-camera detector with a $200 \mu \mathrm{m}$ thick LiF/ZS scintillator coupled to an Andor iKON M camera. The pixel size was $0.105 \mathrm{~mm}$. We determined the intrinsic point-spread function to have a width of $440 \mu \mathrm{m}$ (FWHM). The sampleCRL entry and CRL exit-detector distances were $d_{1}=0.38 \mathrm{~m}$ and $d_{2}=0.76 \mathrm{~m}$, respectively, while the magnification was determined experimentally to be $M=2.18$.

In the transparent lens limit this leads to $f_{N}=256 \mathrm{~mm}, \mathrm{NA}=$ $1.2 \mathrm{mrad}$ and $\mathrm{FOV}=21.8 \mathrm{~mm}$. Moreover, $N T / f_{N}=0.03$, so we (a)

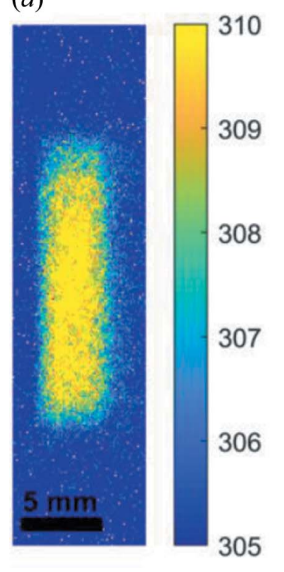

(b)

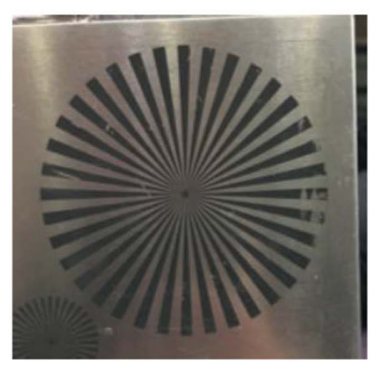

(c)

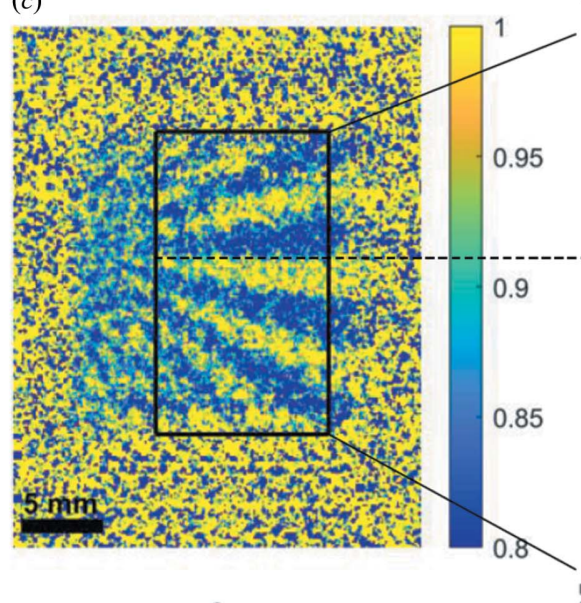

$(d)$

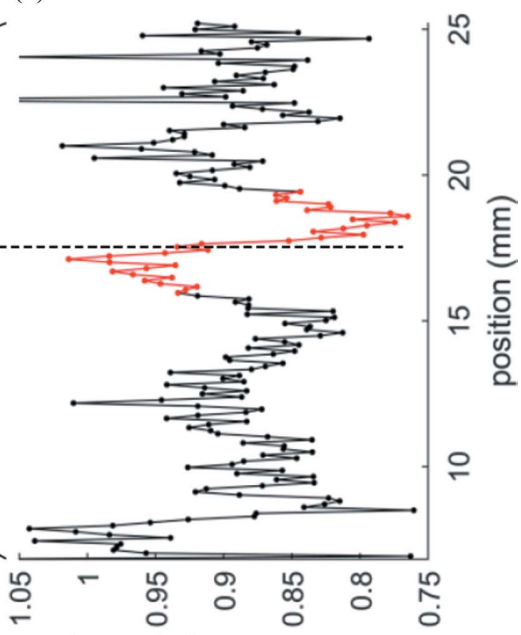

('n'que) Кํ!suәңu! ueәш

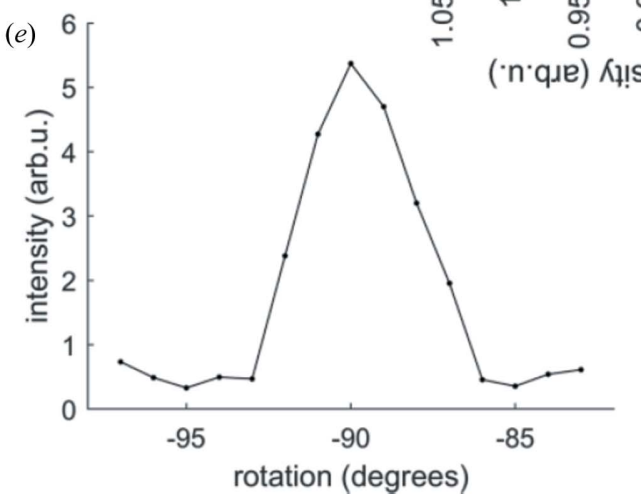

Figure 6

Bright-field microscopy demonstration. (a) A raw image with no sample, with colours representing intensity in arbitrary units. (b) The Siemens Gd startype reference sample. (c) An image of the sample stitched together from individual images acquired while scanning the sample (normalized image with colours indicating intensities in arbitrary units). $(d)$ A vertical intensity profile across spokes. (e) The intensity of the entire image shown as a function of rotation angle around the vertical axis, perpendicular to the incoming beam. 
are clearly in the thin lens limit. The corresponding estimate for the radius of the blurring function associated with chromatic aberration is $r=4.6 \mu \mathrm{m}$.

An image acquired with the sample removed is shown in Fig. 6(a). The resulting field of view in the sample plane is $7.7 \times 2.0 \mathrm{~mm}(\mathrm{FWHM})$. Given the point-spread function of the detector, there is no sign of vignetting in the optical system. Next, the sample was inserted and scanned in the horizontal direction. A resulting stitched image is shown in Fig. 6(c). As expected the image is non-distorted apart from an overall blurring, which appears to be Lorentzian and to be approximately uniform over the image. The FWHM is $0.67 \mathrm{~mm}$. Subtracting the point-spread function of the detector and dividing by the magnification, we reach a rough estimate of the blurring due to the optical setup. The result is a blurring function with a radius of $0.05 \mathrm{~mm}$ in the sample plane.

Next, the sample was rotated around a vertical axis, perpendicular to the beam. The intensity integrated over the entire detector is shown as a function of rotation angle in Fig. 6(e). The width of the intensity profile is $64 \pm 10 \mathrm{mrad}$. In the transparent lens limit this should be compared with a width of $\left[\alpha_{h}^{2}+(2 Y / N T)\right]^{1 / 2}=55 \mathrm{mrad}$.

In summary, the setup could be optimized in several ways. In particular we speculate that the decrease in resolution is due to geometric errors related to the entry and exit holes in the CRL. Nevertheless, the results demonstrate the pertinent features outlined above: the validity of applying the transparent lens limit and the fact that the FoV is much larger than the physical aperture and the resolution.

\section{Discussion}

In contrast with imaging using many other probes, imaging with neutrons today relies almost entirely on lensless techniques. Imaging in a pinhole geometry does provide high flexibility with respect to the field of view and spatial resolution based on the spectrum and beam divergence available. However, neutron imaging has diversified significantly in recent years, both in terms of method and with respect to applications. This diversity, together with successful applications in synchrotron X-ray imaging, has motivated this work where we explore the use of neutron full-field imaging by means of inserting an objective between the sample and the detector. The anticipated low cost and high degree of adaptability have moved us to focus on CRLs, but much of the formalism established and some of the new modalities introduced are also relevant for objectives based on, for example, zone plates or Wolter optics. [For a recent full-field microscopy demonstration experiment using Wolter optics, see Jorba et al. (2019)].

A main shortcoming of refractive optics is the limit on physical aperture and numerical aperture given by the refractive power. We show here that these can in principle be overcome by the introduction of CRL arrays. Another shortcoming is the chromatic aberration. Here we show that, for medium- to low-spatial-resolution work, the loss may be acceptable. The relevance of full-field microscopy as described above is instead whether the science case demands and the available neutron flux supports a large ratio between field of view and spatial resolution. Here the cases of bright field at a distance, SANS and diffraction from large crystals tend to have a flux advantage compared with the case of powder diffraction. Moreover, for bright-field imaging, achromaticity can be ensured by focusing a condenser on the entry plane of the objective.

The gain in FOM with respect to existing instruments will depend strongly on the science case, sample details and details of the current implementation. For these reasons, the gains calculated in the examples above serve only as order-ofmagnitude estimates. In particular, we point out that we have neglected several issues with $\mathrm{S} / \mathrm{N}$ ratio and background noise, as these are dependent on details of detectors and screening.

As a next step we propose to establish whether one or more of the manufacturing routes suggested can be developed to the stage where serial production is feasible, with specifications as presented here. In that connection, a better understanding of the requirement with regard to crystallinity is needed. Polycrystalline lenses with large grains will lead to glitches in the spectrum (Zhang et al., 2019), while nanocrystalline grains will lead to massive secondary extinction and consequently an orders-of-magnitude increase in the effective attenuation. The acceptable range needs to be determined. Another step forward would be full-scale simulations of entire microscopes - with optimized condensers and objectives - using, for example, Monte Carlo methods.

One end goal is to enable CRL-based condensers and objectives to be used to retrofit existing instruments. In that context we mention that for small $Y$ an entire imaging system can be relatively confined. A more ambitious aim is to prepare for a general-purpose neutron microscope, with banks of objectives and detectors covering bright-field, SANS and wide-angle diffraction in one setup.

\section{Conclusions}

The cross sections for thermal neutrons are different from those of visible light, X-rays and electrons, both in terms of magnitude and for the ratio between absorption, coherent scattering and incoherent scattering. At the same time, neutron sources are essentially $4 \pi$ polychromatic sources, in contrast with the heavily collimated and monochromatic beams of lasers or undulator peaks at synchrotrons, and electron microscopes. In this paper we have explored the virtue of transferring well established optical components and imaging systems for full-field microscopy with refractive elements from these other fields to neutron imaging. Our findings are as follows:

(i) A geometric optics description in terms of a 'transparent lens' is in general adequate for a coarse design.

(ii) The accuracy of simple manufacturing methods such as machining is believed to be sufficient for a spatial resolution of $20 \mu \mathrm{m}$ and up. This in connection with the high ratio of $\delta / \mu$ allows for complicated optical designs providing large coverage in direct space or angular space. 
(iii) For a single CRL and at $4 \AA$, the numerical aperture is limited by the refractive power to be around $10 \mathrm{mrad}$, while the physical aperture is below $1 \mathrm{~cm}$. The FOV on the other hand can be much larger than the physical aperture. We suggest that NA and $2 Y$ can be improved by construction of kinoform lenses and/or CRL arrays.

(iv) In comparison with existing neutron imaging instruments, the FOM is typically degraded in terms of angular acceptance and energy bandwidth when introducing a CRL objective. Nevertheless, we have illustrated that, for a range of medium- to low-spatial-resolution applications, a resulting gain in FOM by several orders of magnitude is possible, due to the simultaneous mapping of the properties in many parts of the sample. The simultaneous registration is also seen as an asset in itself for time-resolved studies.

(v) For diffraction-based imaging, an objective-based solution has the fundamental advantage that spatial and angular degrees of freedom decouple. Similar to work in a transmission electron microscope, one may use slits in the back focal plane to define a specific volume in reciprocal space and then provide imaging corresponding to this $Q$-space contrast.

(vi) For bright-field imaging, an objective-based solution has the advantage that an image is provided of a specific plane, the sample plane. This may be used to avoid or access phase contrast. Moreover, the numerical aperture is sufficiently large that depth-resolved imaging seems realistic, with a resolution of a few millimetres.

(vii) For bright-field imaging, partial correction of the chromatic aberrations increases the maximum bandwidth usable without degrading the image, thus improving the efficiency of the imaging setup.

\section{Acknowledgements}

We thank PSI for the beamtime on BOA, ESRF for providing the Be lenslets, Emmanouela Rantsiou for help with the setup at PSI, Pavel Trtik for lending us the Siemens star sample, Christian David and Nikolaj Zangenberg for scientific discussions, and Peter Willendrup for help with the McStas simulations.

\section{Funding information}

Funding for this research was provided by The Innovation Fund of Denmark (award No. 7039-00030B to Hanna Leemreize).

\section{References}

Allman, B., McMahon, P., Nugent, K. A., Paganin, D., Jacobson, D., Arif, M. \& Werner, S. A. (2000). Nature, 408, 158-159.

Altissimo, M. (2004). Microelectron. Eng. 73-74, 644-650.

Beguiristain, H. R., Anderson, I. S., Dewhurst, C. D., Piestrup, M. A., Cremer, J. T. \& Pantell, R. H. (2002). Appl. Phys. Lett. 81, 42904292.

Byelov, D. V., Meijer, J.-M., Snigireva, I., Snigirev, A., Rossi, L., van den Pol, E., Kuijk, A., Philipse, A., Imhof, A., van Blaaderen, A.,
Vroege, G. J. \& Petukhov, A. V. (2013). RSC Adv. 3, 1567015677.

Cereser, A., Strobl, M., Hall, S. A., Steuwer, A., Kiyanagi, R., Tremsin, A. S., Knudsen, E. B., Shinohara, T., Willendrup, P. K., da Silva Fanta, A. B., Iyengar, S., Larsen, P. M., Hanashima, T., Moyoshi, T., Kadletz, P. M., Krooß, P., Niendorf, T., Sales, M., Schmahl, W. W. \& Schmidt, S. (2017). Sci. Rep. 7, 9561.

Chen, H., Downing, R. G., Mildner, D. F. R., Gibson, W. M., Kumakhov, M. A., Ponomarev, I. Y. \& Gubarev, M. V. (1992). Nature, 357, 391-393.

Cremer, J. (2012). Adv. Imaging Electron. Phys. 172, 335-427.

Cremer, J. T., Piestrup, M. A., Park, H., Gary, C. K., Pantell, R. H., Glinka, C. J. \& Barker, J. G. (2005). Appl. Phys. Lett. 87, 161913.

Dewhurst, C. D. \& Grillo, I. (2016). J. Appl. Cryst. 49, 736-742.

Eskildsen, M. R., Gammel, P. L., Isaacs, E. D., Detlefs, C., Mortensen, K. \& Bishop, D. J. (1998). Nature, 391, 563-566.

Falch, K., Detlefs, C., Di Michiel, M., Snigireva, I., Snigirev, A. \& Mathiesen, R. H. (2016). Appl. Phys. Lett. 109, 054103.

Fenske, J., Rouijaa, M., Šaroun, J., Kampmann, R., Staron, P., Nowak, G., Pilch, J., Beran, P., Šittner, P., Strunz, P., Brokmeier, H.-G., Ryukhtin, V., Kadeřávek, L., Strobl, M., Müller, M., Lukáš, P. \& Schreyer, A. (2016). J. Phys. Conf. Ser. 746, 012009.

Gähler, R., Kalus, J. \& Mampe, W. (1980). J. Phys. E Sci. Instrum. 13, 546-548.

Gourrier, A., Wagermaier, W., Burghammer, M., Lammie, D., Gupta, H. S., Fratzl, P., Riekel, C., Wess, T. J. \& Paris, O. (2007). J. Appl. Cryst. 40, s78-s82.

Hofmann, A., Gan, W. M., Rebelo-Kornmeier, J. \& Schöbel, M. (2013). Neutron News, 24(3), 14-17.

Hofmann, M., Schneider, R., Seidl, G., Rebelo-Kornmeier, J., Wimpory, R., Garbe, R. \& Brokmeier, H.-G. (2006). Physica B, 385-386, 1035-1037.

Jorba, P., Schulz, M., Hussey, D., Abir, M., Seifert, M., Tsurkan, V., Loidl, A., Pfleiderer, C. \& Khaykovich, B. (2019). J. Magn. Magn. Mater. 475, 176-183.

Jordan, J. A. Jr, Hirsch, P. M., Lesem, L. B. \& Van Rooy, D. L. (1970). Appl. Opt. 9, 1883-1887.

Kallmann, H. (1948). Research, 1, 254-260.

Kardjilov, N., Manke, I., Strobl, M., Hilger, A., Treimer, W., Meissner, M., Krist, T. \& Banhart, J. (2008). Nat. Phys. 4, 399-403.

Kardjilov, N., Manke, I., Woracek, R., Hilger, A. \& Banhart, J. (2018). Mater. Today, 21, 652-672.

Lehmann, E., Peetermans, S. \& Betz, B. (2015). Neutron News, 26(2), 6-10.

Lehmann, E. H., Peetermans, S., Josic, L., Leber, H. \& van Swygenhoven, H. (2014). Nucl. Instrum. Methods Phys. Res. A, 735, 102-109.

Liebi, M., Georgiadis, M., Kohlbrecher, J., Holler, M., Raabe, J., Usov, I., Menzel, A., Schneider, P., Bunk, O. \& Guizar-Sicairos, M. (2018). Acta Cryst. A74, 12-24.

Liebi, M., Georgiadis, M., Menzel, A., Schneider, P., Kohlbrecher, J., Bunk, O. \& Guizar-Sicairos, M. (2015). Nature, 527, 349-352.

Mildner, D. F. R. \& Gubarev, M. V. (2011). Nucl. Instrum. Methods Phys. Res. A, 634, S7-S11.

Paganin, D., Mayo, S., Gureyev, T., Miller, P. \& Wilkins, S. W. (2002). J. Microsc. 206, 33-40.

Paganin, D., Sales, M., Beltran, M. A., Poulsen, H. F. \& Schmidt, S. (2019). arXiv: 1909.11186.

Peetermans, S., King, A., Ludwig, W., Reischig, P. \& Lehmann, E. H. (2014). Analyst, 139, 5767-5771.

Penfold, J. \& Tucker, I. (2007). J. Phys. Chem. B, 111, 9496-9503.

Pirling, T. (2011). Proc. Eng. 10, 2147-2152.

Poulsen, H. F., Cook, P. K., Leemreize, H., Pedersen, A. F., Yildirim, C., Kutsal, M., Jakobsen, A. C., Trujillo, J. X., Ormstrup, J. \& Detlefs, C. (2018). J. Appl. Cryst. 51, 1428-1436.

Poulsen, H. F., Jakobsen, A. C., Simons, H., Ahl, S. R., Cook, P. K. \& Detlefs, C. (2017). J. Appl. Cryst. 50, 1441-1456. 
Poulsen, S. O., Poulsen, H. F. \& Bentley, P. M. (2014). Nucl. Instrum. Methods Phys. Res. A, 767, 415-420.

Sales, M., Shinohara, T., Sørensen, M. K., Knudsen, E. B., Tremsin, A., Strobl, M. \& Schmidt, S. (2019). J. Phys. D Appl. Phys. 52, 205001.

Santisteban, J. R., Daymond, M. R., James, J. A. \& Edwards, L. (2006). J. Appl. Cryst. 39, 812-825.

Santisteban, J. R., Edwards, L., Steuwer, A. \& Withers, P. J. (2001). J. Appl. Cryst. 34, 289-297.

Schulz, M., Neubauer, A., Masalovich, S., Mühlbauer, M., Calzada, E., Schillinger, B., Pfleiderer, C. \& Böni, P. (2010). J. Phys. Conf. Ser. 211, 012025.

Sears, V. F. (1992). Neutron News, 3(3), 26-37.

Simons, H., Ahl, S. R., Poulsen, H. F. \& Detlefs, C. (2017). J. Synchrotron Rad. 24, 392-401.

Simons, H., Haugen, A. B., Jakobsen, A. C., Schmidt, S., Stöhr, F., Majkut, M., Detlefs, C., Daniels, J. E., Damjanovic, D. \& Poulsen, H. F. (2018). Nat. Mater. 17, 814-819.

Simons, H., King, A., Ludwig, W., Detlefs, C., Pantleon, W., Schmidt, S., Stöhr, F., Snigireva, I., Snigirev, A. \& Poulsen, H. F. (2015). Nat. Commun. 6, 6098.

Snigirev, A., Kohn, V. G., Snigireva, I. I. \& Lengeler, B. (1996). Nature, 384, 49-51.

Sørensen, H. O., Schmidt, S., Wright, J. P., Vaughan, G. B. M., Techert, S., Garman, E. F., Oddershede, J., Davaasambuu, J., Paithankar, K. S., Gundlach, C. \& Poulsen, H. F. (2012). Z. Kristallogr. 227, 6378.

Steuwer, A., Santisteban, J. R., Withers, P. J. \& Edwards, L. (2004). Physica B, 350, 159-161.
Strobl, M., Harti, R., Gruenzweig, C., Woracek, R. \& Plomp, J. (2017). J. Imaging, $\mathbf{3}, 64$.

Strobl, M., Heimonen, H., Schmidt, S., Sales, M., Kardjilov, N., Hilger, A., Manke, I., Shinohara, T. \& Valsecchi, J. (2019). J. Phys. D Appl. Phys. 52, 123001.

Strobl, M., Manke, I., Kardjilov, N., Hilger, A., Dawson, M. \& Banhart, J. (2009). J. Phys. D Appl. Phys. 42, 243001.

Strobl, M., Treimer, W., Kardjilov, N., Hilger, A. \& Zabler, S. (2008). Nucl. Instrum. Methods Phys. Res. B, 266, 181-186.

Suzuki, J., Oku, T., Adachi, T., Shimizu, H. M., Kiyanagi, Y., Kamiyama, T., Hiraga, F., Iwasa, H., Sato, K. \& Furusaka, M. (2004). Nucl. Instrum. Methods Phys. Res. A, 529, 120-124.

Tiziani, H. J. \& Uhde, H. M. (1994). Appl. Opt. 33, 567-572.

Treimer, W. (2019). Neutron Radiography and Tomography. Handbook of Advanced Non-destructive Evaluation, edited by N. Ida \& N. Meyendorf, pp. 1-85. Heidelberg: Springer.

Wang, X., Holden, T., Stoica, A., An, K., Skorpenske, H., Jones, A., Rennich, G. \& Iverson, E. (2010). Mater. Sci. Forum, 652, 105-110.

Willendrup, P., Farhi, E., Knudsen, E., Filges, U. \& Lefmann, K. (2014). J. Neutron Res. 17, 35-43.

Willendrup, P. \& Lefmann, K. (2019). J. Neutron Res. https://doi.org/ 10.3233/JNR-190108.

Woracek, R., Penumadu, D., Kardjilov, N., Hilger, A., Boin, M., Banhart, J. \& Manke, I. (2014). Adv. Mater. 26, 4069-4073.

Woracek, R., Santisteban, J., Fedrigo, A. \& Strobl, M. (2018). Nucl. Instrum. Methods Phys. Res. A, 878, 141-158.

Zhang, Q., Polikarpov, M., Klimova, N., Larsen, H. B., Mathiesen, R., Emerich, H., Thorkildsen, G., Snigireva, I. \& Snigirev, A. (2019). J. Synchrotron Rad. 26, 109-118. 